INFNCA-TH9610

june 1996

\title{
THE DUALITIES OF 3D DILATON GRAVITY
}

\author{
Mariano Cadoni \\ Dipartimento di Scienze Fisiche, Università di Cagliari, \\ Via Ospedale 72, I-09100 Cagliari, Italy. \\ and \\ INFN, Sezione di Cagliari.
}

\begin{abstract}
We investigate Brans-Dicke dilaton gravity theories in $2+1$ dimensions. We show that the reduced field equations for solutions with diagonal metric and depending only on one spacetime coordinate have a continuous $O(2)$ symmetry. Using this symmetry we derive general static and cosmological solutions of the theory. The action of the discrete group $O(2, \mathbb{Z})$ on the space of the solutions is discussed. Three-dimensional string effective theory and three-dimensional general relativity are discussed in detail. In particular, we find that the previously discovered black string solution is dual to a spacetime with a conical singularity.
\end{abstract}

PACS: 04.60.kz, 04.70.-s, 11.25.-Pm 


\section{Introduction}

Target space duality is a very useful concept in string theory [1]. It relates one with the other exact string backgrounds, which from the field theoretical point of view seem to be drastically different. For instance, the duality symmetry interchanges singularities with horizons [2, 3] or, for cosmological string backgrounds [4, 5, 6], it relates expanding universes with contracting ones.

Though duality symmetry is a genuine string feature that cannot be fully understood in terms of a purely (field theoretical) low-energy string theory, one can also analyze it in the context of a string effective theory. In this framework, the duality symmetries have a natural interpretation in terms of discrete symmetry transformations acting on the field theoretical degrees of freedom. This approach has been very useful because, on one hand, one can establish connections between well-known field theoretical dualities and target space duality (for instance the electric-magnetic duality of four dimensional vector fields). On the other hand, one can generalize the concept of duality symmetry to theories whose solutions do not have a direct interpretation in terms of exact string backgrounds, i.e as two-dimensional conformal field theories. For instance, it has been shown that the duality symmetries of the two-dimensional (2D) exact string black hole or cosmological backgrounds have a natural generalization in terms of the symmetries of black hole and cosmological solutions of 2D dilaton gravity models [7, 8]. Moreover, it has been found that scale factor duality is a general feature of isotropic and homogeneous cosmological solutions of $d$-dimensional BransDicke dilaton gravity theories [9].

The purpose of this paper is to analyze, from the point of view of duality symmetries, three-dimensional (3D) Brans-Dicke dilaton gravity theories. This class of models contains, as particular cases, the two most popular models of gravity in three dimensions: 3D general relativity with a cosmological constant, whose black hole solutions were found by Bañados, Teitelboim and Zanelli (BTZ) [10] and 3D string effective theory.

Three-dimensional dilaton gravity has been already studied by several authors [11, 12, 13]. Even though the black hole solutions of the theory have been already found, a general description of its static and cosmological solutions is still lacking. In this paper we first show that for a metric with a diagonal form the requirement of independence of the solutions from two spacetime coordinates implies the existence of a continuous $O(2)$ symmetry of the reduced field equations. In particular, this is true for static, circularly symmetric, solutions and for homogeneous (i.e. space-independent) cosmological solutions. The $O(2)$ symmetry implies the existence of a discrete $O(2, \mathbb{Z})$ symmetry group acting on the space of the solutions, which can be considered as a subgroup of the $O(2,2, \mathbb{Z})$ group. This discrete group appears in the context of string theory both as duality symmetry for $2 \mathrm{D}$ toroidal compactifications and as symmetry of a curved, exact 3D string background independent of two spacetime coordinates (see for example Ref. [1] and references therein). The existence of the continuous $O(2)$ symmetry is related to the solubility of the field equations. In fact, we will be able to find the most general static, circularly symmetric and homogeneous cosmological solutions of our 3D dilaton gravity models. As a by-product we will also obtain the general solutions of this kind for 3D string effective theory and we will find that a duality transformation maps the previously discovered black string 14 into a 3D spacetime with a conical singularity. 
The outline of our paper is as follows. The continuous $O(2)$ and the discrete $O(2, Z Z)$ symmetries of 3D dilaton gravity are derived and described in section 2 . The static, circularly symmetric, solutions of the theory are derived and discussed in section 3, where in particular the action of the duality transformations on the spectrum of the solutions and 3D string effective theory are analyzed. In section 4 we consider cosmological solutions; the general solution together with some interesting particular cases is derived and discussed. Finally, section 5 illustrates our conclusions.

\section{The duality transformations}

Let us consider Brans-Dicke dilaton gravity in $2+1$ dimensions. The action has the following form:

$$
S=\frac{1}{2 \pi} \int d^{3} x \sqrt{-g} e^{-2 \phi}\left(R-4 \omega(\nabla \phi)^{2}+\lambda^{2}\right),
$$

where $R$ is the scalar curvature, $\phi$ is the dilaton, $\lambda$ is a constant and $\omega$ is the (3D) Brans-Dicke parameter. We will trade $\omega$ for a new parameter $k$,

$$
\omega=\left(\frac{1}{k}-1\right)
$$

to make our formulae simpler. To each value of $k$ corresponds a different dilaton gravity theory. For example, $k=\infty$ corresponds to the 3D low-energy string effective action, for $k=0$ one gets $3 \mathrm{D}$ general relativity with a cosmological constant.

The field equations stemming from the action (11), for $k \neq-1$, can be written after some manipulation as follows:

$$
\begin{gathered}
R_{\mu \nu}+2 \nabla_{\mu} \nabla_{\nu} \phi-\frac{4}{k} \partial_{\mu} \phi \partial_{\nu} \phi+\frac{\lambda^{2}}{k+1} g_{\mu \nu}=0, \\
\nabla^{2} e^{-2 \phi}=\frac{k}{k+1} \lambda^{2} e^{-2 \phi} .
\end{gathered}
$$

For $k=-1$ consistency of the field equations requires $\lambda=0$. In the following we will take $k \neq-1$.

In this paper, we will consider only those solutions of the field equations for which the 3D spacetime metric has a diagonal form and that depend only on one spacetime coordinate. This is the case of static, circularly symmetric, solutions. Using the gauge freedom relative to transformations of the radial coordinate, we can write the most general static, circularly symmetric, solution in the following form:

$$
d s^{2}=-e^{2 \rho(\sigma)} d t^{2}+d \sigma^{2}+e^{2 \nu(\sigma)} d \varphi^{2}, \quad 0 \leq \varphi \leq 2 \pi, \quad \phi=\phi(\sigma) .
$$

With this ansatz, the field equations (2) become

$$
\begin{aligned}
& \nu^{\prime \prime}+\nu^{\prime 2}+\nu^{\prime} \rho^{\prime}-2 \nu^{\prime} \phi^{\prime}-\frac{\lambda^{2}}{k+1}=0, \\
& \rho^{\prime \prime}+\rho^{\prime 2}+\nu^{\prime} \rho^{\prime}-2 \rho^{\prime} \phi^{\prime}-\frac{\lambda^{2}}{k+1}=0
\end{aligned}
$$




$$
\begin{aligned}
& \nu^{\prime \prime}+\rho^{\prime \prime}+\nu^{\prime 2}+\rho^{\prime 2}-2 \phi^{\prime \prime}+\frac{4}{k} \phi^{2}-\frac{\lambda^{2}}{k+1}=0, \\
& \phi^{\prime \prime}-\phi^{\prime}\left(2 \phi^{\prime}-\nu^{\prime}-\rho^{\prime}\right)=-\lambda^{2} \frac{k}{2(k+1)},
\end{aligned}
$$

where $^{\prime}=d / d \sigma$. The field equations (4) are invariant under the action of a $O(2)$ group transforming the fields $\rho, \nu, \phi$. To show this assertion, we first note that the equations (田) are equivalent to the equations of motion obtained from the action

$$
S=-\int d \sigma e^{-2 \phi+\rho+\nu}\left[4 \rho^{\prime} \phi^{\prime}+4 \nu^{\prime} \phi^{\prime}-2 \nu^{\prime} \rho^{\prime}+4\left(\frac{1}{k}-1\right) \phi^{2}-\lambda^{2}\right],
$$

by varying $S$ with respect to $\rho, \nu$ and $\phi$, together with the Hamiltonian constraint

$$
4 \rho^{\prime} \phi^{\prime}+4 \nu^{\prime} \phi^{\prime}-2 \nu^{\prime} \rho^{\prime}+4\left(\frac{1}{k}-1\right) \phi^{2}+\lambda^{2}=0
$$

The lagrangian in eq. (5) can be obtained by substituting the ansatz (3) into the action (1), after dropping the total derivative terms, which do not contribute to the equations of motion. For $k \neq-2$ let us define the new variables

$$
\begin{aligned}
Z & =\sqrt{\frac{k+2}{k}} \tilde{Z}+\frac{2}{\sqrt{k(k+2)}} U, \\
\tilde{Z} & =\nu+\rho, \quad Y=\nu-\rho, \\
U & =2 \phi-\nu-\rho .
\end{aligned}
$$

Expressed in terms of $Z, Y, U$, the action (5) and the constraint (6) become respectively

$$
\begin{gathered}
S=\int d \sigma e^{-U}\left[\frac{k+1}{k+2} U^{\prime 2}-\frac{1}{2}\left(Z^{\prime 2}+Y^{\prime 2}\right)+\lambda^{2}\right], \\
\frac{k+1}{k+2} U^{\prime 2}-\frac{1}{2}\left(Z^{\prime 2}+Y^{\prime 2}\right)-\lambda^{2}=0 .
\end{gathered}
$$

It is now easy to see that the action (8) and the constraint (9) are invariant under transformations of the group $O(2)$, which leave $U$ invariant and act on the variables $Z$ and $Y$. This group contains, as subgroups, the rotations in the $(Z, Y)$ plane:

$$
\left(\begin{array}{l}
Z \\
Y
\end{array}\right) \rightarrow\left(\begin{array}{cc}
\cos \beta & -\sin \beta \\
\sin \beta & \cos \beta
\end{array}\right)\left(\begin{array}{l}
Z \\
Y
\end{array}\right)
$$

with $0 \leq \beta \leq 2 \pi$, and the two inversions

$$
\begin{array}{cc}
Y \rightarrow-Y & Y \rightarrow Y \\
Z \rightarrow Z & , \quad Z \rightarrow-Z
\end{array}
$$

We are particularly interested in the discrete $O(2, \mathbb{Z})$ subgroup of the $O(2)$ group. This discrete symmetry of the field equations (田) is clearly related to the $O(2,2, \mathbb{Z})$ symmetry of the exact 3D string backgrounds independent of two spacetime coordinates (see Ref. [1] and 
references therein). As we shall see later, when we will discuss 3D string effective theory, this $O(2, \mathbb{Z})$ symmetry group should be considered as the generalization of 3D target space string duality symmetry to the generic dilaton gravity theory defined by the action (11).

The $O(2, \mathbb{Z})$ group is generated by five elements, the two inversions (11) and three rotations (10) with $\beta=\pi / 2, \pi, 3 \pi / 2$. Using eqs. (7), (10) and (11) one can easily obtain the representation of the $O(2, \mathbb{Z})$ group in terms of transformations of the variables $\rho, \nu, \phi$. We will give here only the representations for the two inversions and the $\beta=\pi$ rotation. These transformations represent three independent subgroups of the $O(2, Z Z)$ group; each transformation $T$ satisfies $T^{2}=I$, where $I$ is the identity. They can be therefore considered as duality transformations of the field equations that, as we will see in the next section, interchange different solutions of the theory. The $\beta=\pi$ rotation and the two inversions (11) are represented, respectively, by the following transformations:

$$
\begin{aligned}
\rho & \rightarrow \frac{1}{k+2}(2 \nu-k \rho-4 \phi), \\
\nu & \rightarrow \frac{1}{k+2}(2 \rho-k \nu-4 \phi), \\
\phi & \rightarrow \frac{1}{k+2}(-k \rho-k \nu+(k-2) \phi) ; \\
\quad \rho \rightarrow \nu, & \rightarrow \phi ; \\
\rho \rightarrow & \frac{1}{k+2}(2 \rho-k \nu-4 \phi), \\
\nu & \rightarrow \frac{1}{k+2}(2 \nu-k \rho-4 \phi), \\
\phi & \rightarrow \frac{1}{k+2}(-k \rho-k \nu+(k-2) \phi) .
\end{aligned}
$$

The previous discussion applies for every $k \neq-2$. It is also interesting to work out in detail the particular cases $k=0$ and $k=\infty$. For $k=0$ the action (11) reduces to 3D general relativity with a cosmological constant. Here the dilaton must be a constant, which can be set equal to zero without loss of generality. The field equations are now invariant only under the inversion $Y \rightarrow-Y$, the duality (13) with $\phi=0$. The $k=\infty$ case corresponds to 3D low-energy string effective theory so that one would expect the dualities (12), (13), (14) to coincide with the 3D target space dualities of string theory. For $k=\infty$ the eqs. (8), (9) become

$$
\begin{aligned}
S & =\int d \sigma e^{-U}\left[U^{\prime 2}-\frac{1}{2}\left(Z^{\prime 2}+Y^{\prime 2}\right)+\lambda^{2}\right], \\
U^{\prime 2} & -\frac{1}{2}\left(Z^{\prime 2}+Y^{\prime 2}\right)-\lambda^{2}=0,
\end{aligned}
$$


whereas the five elements of the $O(2, \mathbb{Z})$ group are represented, in terms of transformations acting on the fields $\rho, \nu, \phi$, as follows

$$
\begin{aligned}
& \rho \rightarrow-\rho \quad \rho \rightarrow \nu \quad \rho \rightarrow-\nu \\
& \nu \rightarrow-\nu \quad, \quad \nu \rightarrow \rho, \quad \nu \rightarrow-\rho, \\
& \phi \rightarrow \phi-\nu-\rho \quad \phi \rightarrow \phi \quad \phi \rightarrow \phi-\nu-\rho \\
& \rho \rightarrow-\nu \quad \rho \rightarrow \nu \\
& \nu \rightarrow \rho \quad, \quad \nu \rightarrow-\rho \quad \text {. } \\
& \phi \rightarrow \phi-\nu \quad \phi \rightarrow \phi-\rho
\end{aligned}
$$

The transformations (15) are the dualities of the field equations (4) in the $k=\infty$ case and can be obtained by taking this limit in eqs. (12), (13), (14). The transformations (16) represent the $\beta=\pi / 2,3 \pi / 2$ rotations of the $O(2, Z Z)$ group. In the next section we will discuss in detail the action of these duality transformations on the space of the solutions of the field equations.

Until now our discussion has been confined to 3D solutions of the form (3). However, it is not difficult to realize that our derivation holds in every situation where the metric has a diagonal form and the solutions depend only on one spacetime coordinate. In particular our discussion applies to time-dependent solutions of the form:

$$
d s^{2}=-d t^{2}+e^{2 \rho(t)} d \chi^{2}+e^{2 \nu(t)} d \varphi^{2}, \quad 0 \leq \varphi \leq 2 \pi, \quad 0 \leq \chi \leq 2 \pi, \quad \phi=\phi(t) .
$$

Notice that we consider 3D cosmological solutions with two compact spatial dimensions. All the features of the model described in this section rely only on the diagonal form of the metric and on its independence from two of the three spacetime coordinates, so that the generalization to a spacetime with metric (17) is straightforward.

Using the ansatz (17) the field equations (2) become

$$
\begin{aligned}
& \ddot{\nu}+\dot{\nu}^{2}+\dot{\nu} \dot{\rho}-2 \dot{\nu} \dot{\phi}+\frac{\lambda^{2}}{k+1}=0, \\
& \ddot{\rho}+\dot{\rho}^{2}+\dot{\nu} \dot{\rho}-2 \dot{\rho} \dot{\phi}+\frac{\lambda^{2}}{k+1}=0, \\
& \ddot{\nu}+\ddot{\rho}+\dot{\nu}^{2}+\dot{\rho}^{2}-2 \ddot{\phi}+\frac{4}{k} \dot{\phi}^{2}+\frac{\lambda^{2}}{k+1}=0, \\
& \ddot{\phi} \quad-\dot{\phi}(2 \dot{\phi}-\dot{\nu}-\dot{\rho})=\lambda^{2} \frac{k}{2(k+1)},
\end{aligned}
$$

where the dots denote the derivative $d / d t$. Again, these equations are equivalent to the equations of motion derived from the action

$$
S=\int d t e^{-2 \phi+\rho+\nu}\left(4 \dot{\rho} \dot{\phi}+4 \dot{\nu} \dot{\phi}-2 \dot{\nu} \dot{\rho}+4\left(\frac{1}{k}-1\right) \dot{\phi}^{2}+\lambda^{2}\right),
$$

together with the Hamiltonian constraint

$$
4 \dot{\nu} \dot{\phi}+4 \dot{\phi} \dot{\rho}-2 \dot{\rho} \dot{\phi}+4\left(\frac{1}{k}-1\right) \dot{\phi}^{2}-\lambda^{2}=0 .
$$


Using the redefinitions (7), the previous equations can be written in terms of the new variables $U, Z, Y$ as follows

$$
\begin{gathered}
S=-\int d t e^{-U}\left[\frac{k+1}{k+2} \dot{U}^{2}-\frac{1}{2}\left(\dot{Z}^{2}+\dot{Y}^{2}\right)-\lambda^{2}\right], \\
\frac{k+1}{k+2} \dot{U}^{2}-\frac{1}{2}\left(\dot{Z}^{2}+\dot{Y}^{2}\right)+\lambda^{2}=0 .
\end{gathered}
$$

The continuous $O(2)$ symmetry (10), (11]) and the duality symmetries (12), (13), (14) of the field equations, discussed previously for the static solution (3), are also symmetries of the cosmological solutions (17). The duality symmetries of these solutions are now a generalization to the generic dilaton gravity theory defined by the action (1) of the scale factor duality [4, 5, 6] of low-energy string theory .

\section{Static solutions}

In this section we will derive and discuss the most general static, circularly symmetric, solution of our 3D dilaton gravity theory. In particular, we will also analyze the action of the dualities (12), (13), (14) on the space of the solutions.

The continuous $O(2)$ symmetry is a feature of the field equations (4) that is strongly related with their solubility. In fact, the field redefinitions (7) not only make the $O(2)$ symmetry explicit but also enable us to write the field equations (4) in a form that makes them elementary solvable. Varying the action (8) with respect to $Z, Y, U$ and using the constraint (9), one gets

$$
\begin{aligned}
& Z^{\prime \prime}-U^{\prime} Z^{\prime}=0, \\
& Y^{\prime \prime}-U^{\prime} Y^{\prime}=0, \\
& U^{\prime \prime}-U^{\prime 2}+\frac{k+2}{k+1} \lambda^{2}=0 .
\end{aligned}
$$

These equations, supported with the constraint (9), are equivalent to the field equations (4). The system of differential equations (18) can be easily integrated. The analytic expressions and the properties of the corresponding solutions depend on the value of the parameter $k$, therefore we will discuss the various cases separately.

\section{$3.1\{\mathrm{k}<-2\} \cup\{\mathrm{k}>-1\}$}

The solutions, expressed in terms of the metric variables $\rho, \nu$ and of the dilaton $\phi$, are

$$
\begin{aligned}
e^{2 \rho} & =A\left[\sinh \alpha\left(\sigma-\sigma_{0}\right)\right]^{2 \gamma+\frac{2}{k+2}}\left[\cosh \alpha\left(\sigma-\sigma_{0}\right)\right]^{\frac{2}{k+2}-2 \gamma}, \\
e^{2 \nu} & =B\left[\sinh \alpha\left(\sigma-\sigma_{0}\right)\right]^{2 \delta+\frac{2}{k+2}}\left[\cosh \alpha\left(\sigma-\sigma_{0}\right)\right]^{\frac{2}{k+2}-2 \delta}, \\
e^{-2 \phi} & =C\left[\sinh \alpha\left(\sigma-\sigma_{0}\right)\right]^{-(\gamma+\delta)+\frac{k}{k+2}}\left[\cosh \alpha\left(\sigma-\sigma_{0}\right)\right]^{\gamma+\delta+\frac{k}{k+2}},
\end{aligned}
$$


where

$$
\alpha=\frac{1}{2} \lambda \sqrt{\frac{k+2}{k+1}}
$$

and $A, B, C, \sigma_{0}, \gamma, \delta$ are integration constants. The constants $A, B, C, \sigma_{0}$ are arbitrary. $\sigma_{0}$ is irrelevant for our discussion, in the following we will set $\sigma_{0}=0$. The constants $\gamma$ and $\delta$ are not arbitrary, in fact the constraint (9) implies

$$
\gamma^{2}+\delta^{2}+\frac{2}{k+1} \gamma \delta=\frac{k}{k+2}
$$

The solutions (19) describe black holes with a regular horizon only for

$$
\gamma=\frac{k+1}{k+2}, \quad \delta=-\frac{1}{k+2} .
$$

In this case the coordinate transformation

$$
r=\left(\frac{b}{a^{2}}\right)^{\frac{1}{k+2}}(\cosh \alpha \sigma)^{\frac{2}{k+2}}, \quad a^{2}=\frac{\lambda^{2}}{(k+1)(k+2)},
$$

with an appropriate choice of the constants $A, B$ and $C$, brings the solutions (19) into the following form:

$$
\begin{aligned}
d s^{2} & =-\left[(a r)^{2}-b r^{-k}\right] d t^{2}+\left[(a r)^{2}-b r^{-k}\right]^{-1} d r^{2}+r^{2} d \varphi^{2}, \\
e^{-2 \phi} & =(a r)^{k}, \quad b \geq 0 .
\end{aligned}
$$

The constant $b$ appearing in the previous equations is related to the Arnowitt-Deser-Misner mass of the black hole by $M=(k+1) a^{k} b$. The solutions (22) are the black hole solutions first found in Ref. [11]. They describe a spacetime with anti-de Sitter asymptotical behavior. For $k>-1$ and $b \neq 0$ we have a curvature singularity, shielded by the event horizon, located at $r=0$, whereas the asymptotical region is at $r=\infty$. For $k<-2$ the curvature singularity is located at $r=\infty$ whereas the asymptotical region is at $r=0$.

The duality transformations (12), (13), (14) act on the space of the solutions relating the black hole solutions (22) to other solutions of the field equations, similarly to what happens in 2D dilaton gravity theories [7]. The duality (12) transforms in eqs. (19) $\gamma \rightarrow$ $-\gamma, \delta \rightarrow-\delta$. This is equivalent to interchange everywhere in eqs. (19) the hyperbolic sine with the hyperbolic cosine. The solution dual to (22) is therefore characterized by $\gamma=-(k+1) /(k+2), \delta=1 /(k+2)$. It can be put in the form (22) (with $b<0$ ) using the coordinate transformation

$$
r=\left(-\frac{b}{a^{2}}\right)^{\frac{1}{k+2}}(\sinh \alpha \sigma)^{\frac{2}{k+2}}
$$

Thus, the duality (12) transforms a black hole (a solution (22) with $b>0$ ) in a spacetime with a naked singularity (a solution (22) with $b<0$ ). The duality (13) interchanges $\rho$ and $\nu$ leaving the dilaton invariant $(\gamma \leftrightarrow \delta$ in eqs. (19)). The black hole solutions (22) are mapped by this duality into the solutions

$$
\begin{aligned}
d s^{2} & =-r^{2} d t^{2}+\left[(a r)^{2}-b r^{-k}\right]^{-1} d r^{2}+\left[(a r)^{2}-b r^{-k}\right] d \varphi^{2}, \\
e^{-2 \phi} & =(a r)^{k}, \quad b \geq 0 .
\end{aligned}
$$


In the neighborhood of $r=r_{0}=\left(b / a^{2}\right)^{1 /(k+2)}$ the line element (23) can be brought into the form

$$
d s^{2}=-d t^{2}+d \sigma^{2}+h^{2} \sigma^{2} d \varphi^{2}
$$

where $h$ is a constant, which depends only on the parameters $a$ and $b . r=r_{0}$ is just a conical singularity for the metric (23). With an appropriate choice of the constant $B$ appearing in eqs. (19), one can have $h=1$, removing the conical singularity, so that the point $r=r_{0}$ can be considered as the origin of the radial coordinate. In this case the solution (23) with $k>-1$ describes a regular spacetime, which is asymptotically anti-de Sitter. It is important to stress that the spacetime with the conical singularity not this regular solution is the dual of the black hole (22).

The duality (14) transforms in eqs. (19) $\gamma \rightarrow-\delta, \delta \rightarrow-\gamma$. This transformation can be obtained from the product of the dualities (12) and (13). It is easy to see that it maps the black hole solutions (22) into the solutions (23) with $b<0$. In this case the solutions describe a spacetime with a naked singularity.

\section{$3.2-2<\mathrm{k}<-1$}

The equations (18) are now solved by the expressions (19) with the hyperbolic functions replaced by the corresponding trigonometric ones and with $\alpha=\frac{\lambda}{2} \sqrt{\left|\frac{k+2}{k+1}\right|}$. The integration constants $\gamma$ and $\delta$ have to satisfy the same constraint (21). The solutions characterized by

$$
\gamma=\frac{k+1}{k+2}, \quad \delta=-\frac{1}{k+2}
$$

now describe a spacetime with a cosmological horizon. In fact performing the coordinate transformation

$$
r=\left(\frac{b}{a^{2}}\right)^{\frac{1}{k+2}}(\cos \alpha \sigma)^{\frac{2}{k+2}}
$$

these solutions become

$$
\begin{aligned}
d s^{2} & =-\left[b r^{-k}-(a r)^{2}\right] d t^{2}+\left[b r^{-k}-(a r)^{2}\right]^{-1} d r^{2}+r^{2} d \varphi^{2} \\
e^{-2 \phi} & =(a r)^{k}, \quad b \geq 0
\end{aligned}
$$

The dualities (12), (13), (14) act on the solution (25) in a way similar to that described in sect. 3.1. One must simply replace everywhere hyperbolic with trigonometric functions. In particular, the duality (12) transforms in (25) $b \rightarrow-b$, i.e the dual of (25) is a singular spacetime with no horizons. The duality (13) transforms the spacetime (25) into

$$
\begin{aligned}
d s^{2} & =-r^{2} d t^{2}+\left[b r^{-k}-(a r)^{2}\right]^{-1} d r^{2}+\left[b r^{-k}-(a r)^{2}\right] d \varphi^{2} \\
e^{-2 \phi} & =(a r)^{k}
\end{aligned}
$$

with $b \geq 0$ whereas (14) transforms (25) into the same solution but with $b \leq 0$. 


\section{$3.3 \mathrm{k}=\infty$}

This case corresponds to 3D string effective theory. The static, circularly symmetric, solutions are obtained by taking in eqs. (19), (20) and (21) (we set there $A=1, B=\lambda^{-2}$ ) the $k \rightarrow \infty$ limit:

$$
\begin{aligned}
e^{2 \rho} & =\left(\tanh \frac{\lambda}{2} \sigma\right)^{2 \gamma} \\
e^{2 \nu} & =\lambda^{-2}\left(\tanh \frac{\lambda}{2} \sigma\right)^{2 \delta}, \\
e^{-2 \phi} & =C\left(\sinh \frac{\lambda}{2} \sigma\right)^{1-(\gamma+\delta)}\left(\cosh \frac{\lambda}{2} \sigma\right)^{\gamma+\delta+1},
\end{aligned}
$$

with $\delta^{2}+\gamma^{2}=1$. The change of variable

$$
\frac{r}{M}=\left(\cosh \frac{\lambda}{2} \sigma\right)^{2}
$$

enables us to write (26) as follows

$$
\begin{aligned}
d s^{2} & =-\left(1-\frac{M}{r}\right)^{\gamma} d t^{2}+\left(1-\frac{M}{r}\right)^{-1} \frac{1}{\lambda^{2} r^{2}} d r^{2}+\lambda^{-2}\left(1-\frac{M}{r}\right)^{\delta} d \varphi^{2}, \\
e^{-2 \phi} & =\lambda r\left(1-\frac{M}{r}\right)^{(1-\gamma-\delta) / 2}, \quad \gamma^{2}+\delta^{2}=1 .
\end{aligned}
$$

Differently from the general case, this solution describes a spacetime that is asymptotically flat with topology $R^{1,1} \times S^{1}$ (we consider here a compact coordinate $\varphi$, our consideration can be easily generalized to the case of a noncompact $\varphi$ ). This implies that string effective theory in three dimensions does not admit black hole but only black string solutions. This result is already known [14, 15] and eq. (27), being the general static, circularly symmetric, solution of 3D string effective theory, gives a straightforward prove of it. The black string of Ref. [14] is obtained from eq. (27) (or equivalently from (26)) by setting there $\gamma=1, \delta=0$ :

$$
\begin{aligned}
d s & =-\left(1-\frac{M}{r}\right) d t^{2}+\left(1-\frac{M}{r}\right)^{-1} \frac{1}{\lambda^{2} r^{2}} d r^{2}+\lambda^{-2} d \varphi^{2}, \\
e^{-2 \phi} & =\lambda r, \quad M \geq 0 .
\end{aligned}
$$

For generic values of the parameters $\gamma$ and $\delta$ the solution (27) presents a curvature singularity at $r=M$. For $\gamma=-1, \delta=0$ the solution has the form (28) but with $M \leq 0$. In this case the spacetime has a curvature singularity at $r=0$ not shielded by an event horizon. For $\gamma=0$ and $\delta= \pm 1$ the solutions can be put into the following form

$$
\begin{aligned}
d s^{2} & =-\lambda^{-2} d t^{2}+\left(1-\frac{M}{r}\right)^{-1} \frac{1}{\lambda^{2} r^{2}} d r^{2}+\left(1-\frac{M}{r}\right) d \varphi^{2}, \\
e^{-2 \phi} & =\lambda r
\end{aligned}
$$


where $M \geq 0$ for $\delta=1$ and $M \leq 0$ for $\delta=-1$. For $M>0, r=M$ is a conical singularity of the metric. In fact, in the neighborhood of $r=M$ the line element (29) reduces to the form (24). We can remove this conical singularity by choosing appropriately either the integration constants or the period of the compact coordinate $\varphi$. Near $r=M$ the spacetime has the local topology of $R^{1,2}$. Thus, $r=M$ can be considered as the origin of a polar coordinate system of the plane. In this case the metric (29) describes therefore a 3D regular spacetime with local topology $R^{1,1} \times S^{1}$ for $r \rightarrow \infty$ and $R^{1,2}$ for $r \rightarrow M$. Conversely, for $M<0$ the line element (29) has a curvature singularity at $r=0$.

As for the general case, using the duality transformations (15) one can relate the black string solution (28) to the various spacetimes discussed in this section. In particular, the first duality in eqs. (15), which interchanges the hyperbolic sine and cosine, is the well-known target space duality of 2D string theory that interchanges the horizon and the singularity of the 2D black hole geometry [1], 2, 3]. This transformation maps in eqs. (27) the solutions with positive $M$ in those with $M$ negative. The second duality in eqs. (15) maps the black string into the spacetime with a conical singularity described by the line element (29) with $M>0$. Finally, the third duality in eqs. (15) relates the black string solution to the line element (29) with $M<0$, which describes a spacetime with a curvature singularity.

\section{$3.4 \mathrm{k}=0,-2$}

For $k=0$, which corresponds to 3D general relativity, the dilaton must be constant and eq. (21) implies $\delta=-\gamma$. Introducing the new radial coordinate $r=\frac{\sqrt{b}}{a} \cosh \alpha \sigma$, the general solution takes the form:

$$
d s^{2}=-(a r)^{1-2 \gamma}\left[(a r)^{2}-b\right]^{\frac{1}{2}+\gamma} d t^{2}+\left[(a r)^{2}-b\right]^{-1} d r^{2}+(a r)^{1+2 \gamma}\left[(a r)^{2}-b\right]^{\frac{1}{2}-\gamma} a^{-2} d \varphi^{2},
$$

where $\gamma$ now is an arbitrary constant. We have regular black holes solutions only for $\gamma=1 / 2$, which gives the BTZ black hole [10]

$$
d s^{2}=-\left[(a r)^{2}-b\right] d t^{2}+\left[(a r)^{2}-b\right]^{-1} d r^{2}+r^{2} d \varphi^{2}
$$

Here the only relevant duality is (13) that interchanges the BTZ black hole solutions with $b>0$ with those with $b<0$.

For $k=-2$ the solutions to the field equations (18) are

$$
\begin{aligned}
e^{2 \rho} & =A \exp \left(\frac{-\lambda^{2} \sigma^{2}}{2}\right)(\lambda \sigma)^{\delta-\gamma}, \\
e^{2 \nu} & =B \exp \left(\frac{-\lambda^{2} \sigma^{2}}{2}\right)(\lambda \sigma)^{\delta+\gamma}, \\
e^{2 \rho} & =C \exp \left(\frac{\lambda^{2} \sigma^{2}}{2}\right)(\lambda \sigma)^{1-\delta}, \\
\gamma^{2} & +2 \delta-3=0 .
\end{aligned}
$$

For generic values of the parameters $\gamma$ and $\delta$ the corresponding metric has curvature singularities both at $\sigma=0$ and $\sigma=\infty$. For $\delta=1, \gamma= \pm 1, \sigma=0$ becomes a coordinate 
singularity. The duality transformations (12) and (14) become singular for $k=2$. The only transformation that is a symmetry of the field equations is now given by eq. (13). This transformation flips the sign of $\gamma$ in the solutions.

\section{Cosmological solutions}

Let us now consider time-dependent solutions that have the form (17). The field equations and the hamiltonian constraint can be written as follows

$$
\begin{aligned}
\ddot{Z} & -\dot{U} \dot{Z}=0 \\
\ddot{Y} & -\dot{Y} \dot{Z}=0, \\
\ddot{U} & -\dot{U}^{2}-\frac{k+2}{k+1} \lambda^{2}=0 \\
\lambda^{2} & +\frac{k+1}{k+2} \dot{U}^{2}-\frac{1}{2}\left(\dot{Z}^{2}+\dot{Y}^{2}\right)=0
\end{aligned}
$$

This system of differential equations can be easily solved. In fact, it is related to the static case (eqs.(18)) by the substitution $\sigma \rightarrow i t$. In the same way as for the static solutions, we will consider separately the models with different values of the parameter $k$.

\section{1 $\{\mathrm{k}<-2\} \cup\{\mathrm{k}>-1\}$}

Expressed in terms of the metric functions $\rho, \nu$ and of the dilaton $\phi$, the general solution of the eqs. (30) is

$$
\begin{aligned}
e^{2 \rho} & =A\left[\sin \alpha\left(t-t_{0}\right)\right]^{2 \gamma+\frac{2}{k+2}}\left[\cos \alpha\left(t-t_{0}\right)\right]^{\frac{2}{k+2}-2 \gamma}, \\
e^{2 \nu} & =B\left[\sin \alpha\left(t-t_{0}\right)\right]^{2 \delta+\frac{2}{k+2}}\left[\cos \alpha\left(t-t_{0}\right)\right]^{\frac{2}{k+2}-2 \delta}, \\
e^{-2 \phi} & =C\left[\sin \alpha\left(t-t_{0}\right)\right]^{-(\gamma+\delta)+\frac{k}{k+2}}\left[\cos \alpha\left(t-t_{0}\right)\right]^{\gamma+\delta+\frac{k}{k+2}} .
\end{aligned}
$$

In these equations $\alpha$ is given by eq. (20) and the constants $\gamma$ and $\delta$ have to satisfy eq. (21). $A, B, C, t_{0}$ are arbitrary constant, which in the following we will be fixed by setting $A=B=\alpha^{-2}, C=1$ and $t_{0}=0$.

The solutions (31) have the typical form of the string cosmological solutions that have been already discussed in the literature [6, 16, 8]. The surfaces with $t=$ const in the line element (17) have topology $S^{1} \times S^{1}$ and the scale factors $\exp (2 \rho)$ and $\exp (2 \nu)$ have to be considered as the (time-dependent) radii of the two one-spheres $S^{1}$. In general, the scale factors are periodic, with period $2 \pi / \alpha$. For generic values of $\gamma$ and $\delta$ the line element has curvature singularities both at $t=n \pi / \alpha$ and $t=(n+1) \pi / 2 \alpha,(n=0,1,2 .$.$) . For$ $\gamma=(k+1) /(k+2), \delta=-1 /(k+2)$ the solutions (31) become

$$
\begin{aligned}
e^{2 \rho} & =\alpha^{-2}(\sin \alpha t)^{2}(\cos \alpha t)^{-2 k /(k+2)}, \\
e^{2 \nu} & =\alpha^{-2}(\cos \alpha t)^{4 /(k+2)} \\
e^{-2 \phi} & =(\cos \alpha t)^{2 k /(k+2)}
\end{aligned}
$$


Though $t=(n+1) \pi / 2 \alpha$ is still a curvature singularity, $t=n \pi / \alpha$ becomes a coordinate singularity of the metric. In fact, in the neighborhood of this point the line element (17) reduces to the form

$$
d s^{2}=-d t^{2}+t^{2} d \chi^{2}+\alpha^{-2} d \varphi^{2} .
$$

The topology of the spacetime is locally $R^{1,1} \times S^{1}$ and the surface $t=0$ is homotopic to $S^{1}$ and a point. For $\gamma=-1 /(k+2), \delta=(k+1) /(k+2)$ we have the same situation but the scale factors of the two one-spheres are interchanged. The solution has now the following form:

$$
\begin{aligned}
e^{2 \rho} & =\alpha^{-2}(\cos \alpha t)^{4 /(k+2)}, \\
e^{2 \nu} & =\alpha^{-2}(\sin \alpha t)^{2}(\cos \alpha t)^{-2 k /(k+2)}, \\
e^{-2 \phi} & =(\cos \alpha t)^{2 k /(k+2)} .
\end{aligned}
$$

The action of the duality transformations (12), (13), (14) on the space of the solutions is similar to that described in sec. 3.1 for the static solutions. In particular, the duality (12) is the 3D analogue of the 2D scale factor duality discussed in Ref. [8]. It interchanges everywhere in eqs. (31) the sine with the cosine, so that for generic $\gamma$ and $\delta$ it interchanges one with the other the two curvature singularities at $t=(n+1) \pi / 2 \alpha$ and $t=n \pi / \alpha$. In the case of solutions (32), (33) it interchanges the curvature singularity at $t=(n+1) \pi / 2 \alpha$ and the coordinate singularity at $t=n \pi / \alpha$. The duality (13) interchanges the scale factor $\exp 2 \rho$ with the scale factor $\exp 2 \nu$ leaving the dilaton invariant. Solution (32) is mapped by this transformation into solution (33) and vice versa. Finally, the duality (14), being the product of dualities (12), (13) first interchanges the sine and the cosine then interchanges the scale factors of the two $S^{1}$.

\section{$4.2-2<\mathrm{k}<-1$}

The solutions to the field equations (30) can now be given in terms of hyperbolic functions

of argument $\alpha$, with $\alpha=(\lambda / 2) \sqrt{|(k+2) /(k+1)|}$. They have the form (31) with the trigonometric functions replaced by the corresponding hyperbolic ones. The main difference between the present case and that of Sect. (4.1) is that now the cosmological solutions are not any more periodic. The generic solution has only a curvature singularity at $t=0$. For the special values of the parameters $\gamma, \delta$ leading to the solutions (32), (33), the curvature singularity at $t=0$ becomes a coordinate singularity. In this case the solutions describe a regular spacetime. All the main features of the solutions described in the previous subsection, concerning the action of the duality transformations on the space of the solutions, can be easily translated to the present case.

\section{$4.3 \mathrm{k}=\infty$}

Performing the limit $k \rightarrow \infty$ in eqs. (31) one obtains the cosmological solutions of 3D string effective theory:

$$
\begin{aligned}
e^{2 \rho} & =\alpha^{-2}(\tan \alpha t)^{2 \gamma} \\
e^{2 \nu} & =\alpha^{-2}(\tan \alpha t)^{2 \delta} \\
e^{-2 \phi} & =(\sin \alpha t)^{-(\gamma+\delta)+1}(\cos \alpha t)^{\gamma+\delta+1}
\end{aligned}
$$


with $\delta^{2}+\gamma^{2}=1$. The metric has the same singularities as in the general case. For generic values of the parameters $\gamma$ and $\delta$ we have two curvature singularities whereas for $\gamma=1, \delta=0$ or $\gamma=0, \delta=1$ the singularity at $t=n \pi / \alpha$ becomes a coordinate singularity. For these values of the parameters $\gamma$ and $\delta$ the radius of one $S^{1}$ is constant and the spacetime has topology $H \times S^{1}$ where $H$ describes the well-known cosmological solution of $2 \mathrm{D}$ string effective theory [16, 6]. Moreover, the solution with $\gamma=1, \delta=0$ is related to that with $\gamma=0, \delta=1$ by the second duality transformation in eqs. (15), which interchanges the scale factors of the two $S^{1}$. The first duality in eqs. (15) is the 3D analogue of the scale factor duality of $2 \mathrm{D}$ string effective theory, to which it reduces when either $\rho$ or $\nu$ is constant. Finally, the third duality in eqs. (15) is the product of the first two.

\section{$4.4 \mathrm{k}=0,-2$}

For $k=0$ the dilaton must be constant and the equations (31) yield

$$
\begin{aligned}
e^{2 \rho} & =\frac{4}{\lambda^{2}}\left(\sin \frac{\lambda}{2} t\right)^{2 \gamma+1}\left(\cos \frac{\lambda}{2} t\right)^{1-2 \gamma}, \\
e^{2 \nu} & =\frac{4}{\lambda^{2}}\left(\sin \frac{\lambda}{2} t\right)^{1-2 \gamma}\left(\cos \frac{\lambda}{2} t\right)^{1+2 \gamma},
\end{aligned}
$$

where $\gamma$ now is an arbitrary integration constant. The previous equations give the cosmological solutions of 3D general relativity with a cosmological constant. For generic $\gamma$ we have, again, two curvature singularities. For $\gamma= \pm 1 / 2$ one of these becomes a coordinate singularity. The dualities (12), (13), (14) now reduce to one single transformation, which transforms $\gamma \rightarrow-\gamma$ or equivalently interchanges the sine and the cosine in the solutions.

For $k=-2$ the solutions to the system (30) can be written as follows

$$
\begin{aligned}
e^{2 \rho}= & \lambda^{-2} \exp \left(\frac{\lambda^{2} t^{2}}{2}\right)(\lambda t)^{\delta-\gamma}, \\
e^{2 \nu}= & \lambda^{-2} \exp \left(\frac{\lambda^{2} t^{2}}{2}\right)(\lambda t)^{\delta+\gamma}, \\
e^{-2 \phi}= & \exp \left(\frac{-\lambda^{2} t^{2}}{2}\right)(\lambda t)^{1-\delta}, \\
& \gamma^{2}+2 \delta-3=0 .
\end{aligned}
$$

For generic $\gamma, \delta$ these solutions have a curvature singularity both at $t=0$ and $t=\infty$. For $\delta=1, \gamma= \pm 1, t=0$ becomes a coordinate singularity. The only transformation that is a duality of the field equations is now given by eq. (13), which flips the sign of $\gamma$ in the solutions.

\section{$5 \quad$ Summary and outlook}

In this paper we have discussed in detail the duality symmetries of 3D dilaton gravity theories. We have found that for spacetime metrics with a diagonal form the independence 
of the solutions from two spacetime coordinates implies the existence of a continuous $O(2)$ and therefore a discrete $O(2, \mathbb{Z})$, symmetry of the reduced field equations. In the case of $3 \mathrm{D}$ string effective theory $(k=\infty)$ this represents a full field theoretical derivation of the discrete $O(2, \mathbb{Z})$ duality symmetry, appearing as subgroup of the target space duality group of 3D string theory. Our derivation, being based on purely field theoretical arguments, holds for 3D Brans-Dicke dilaton gravity theories but it is not clear if it can be generalized to these theories in generic $d$ dimensions. The results of ref. [9] indicate that this is possible at least for homogeneous and isotropic cosmological solutions.

From a more conventional point of view, the existence of duality symmetries made it possible to unfold fully the richness of the spectrum of the solution of 3D dilaton gravity theories. Until now, only few of these solutions were known, mainly the black hole solutions of the generic theory, the black string solution of the string effective theory and other solutions of dilaton gravity coupled to antisymmetric tensor fields. We have been able to write down general static and cosmological solutions of 3D dilaton gravity. Surprisingly enough, we have found that 3D string effective theory has static solutions different from the black string. These solutions describe either a regular spacetime or a spacetime with a conical singularity. Besides, the solutions with the conical singularity are dual to the black string solutions. Presently, we do not know if this is just a result of string effective theory or if it has a counterpart in terms of exact string backgrounds, i.e if these 3D spacetimes with conical singularities have an interpretation as conformal field theories. Exact 3D string backgrounds

with similar conical singularities have been found by Horne and Horowitz in the context of Wess-Zumino-Witten models [14]. These are also solutions of 3D string effective theory with an antisymmetric tensor. We do not know if these solutions with axion charge are related to those we have found in this paper. In order to answer to this question, one should be able to find the general static solution of 3D dilaton gravity coupled to the antisymmetric tensor. We leave this issue to further investigations.

\section{References}

[1] A. Giveon, M. Porrati, E. Rabinovici, Phys. Rep. 244, 77 (1994).

[2] A. Giveon, Mod. Phys. Lett. A6, 2843 (1991).

[3] R. Dijkgraaf, E. Verlinde, , H. Verlinde, Nucl. Phys. B371, 269 (1992).

[4] G. Veneziano, Phys. Lett. B265, 287 (1991).

[5] M. Gasperini and G. Veneziano, Phys. Lett. B277, 265 (1992).

[6] A.A. Tseytlin, C. Vafa, Nucl. Phys. B372, 443 (1992). 
[7] M. Cadoni, S. Mignemi, Mod. Phys. Lett. A10, 367 (1995).

[8] M. Cadoni, M. Cavaglià, Phys. Rev. D50, 6435 (1994).

[9] J.E. Lidsey, "Scale factor duality and hidden supersymmetry in scalar-tensor cosmology", gr-qc/9510017, to appear in Phys. Rev. D15.

[10] M. Bañados, C. Teitelboim, J. Zanelli, Phys. Rev. Lett. 69, 1849 (1992).

[11] P.M. Sá, A. Kleber, J. P. S. Lemos, Class. Quant. Grav. 13,125 (1996).

[12] K.C.K. Chan, R.B. Mann, Phys. Rev. D50, 6385 (1994).

[13] J.P.S. Lemos Phys. Lett. B353, 46 (1995).

[14] J.H. Horne and G.T. Horowitz, Nucl. Phys. B368, 444 (1992).

[15] G.T. Horowitz "The dark side of string theory: black holes and black strings", hepth/9210119.

[16] M. Mueller, Nucl. Phys. B337, 37 (1990). 